\title{
An Abdominal Aortic Aneurysm, Intramural Thrombus and Moderate Leak in an Asian Man Presenting with Acute Gastroenteritis
}

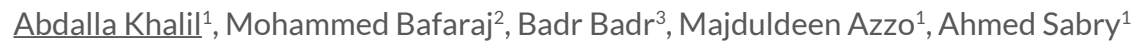 \\ ${ }^{1}$ Emergency Department, IMC Hospital, Jeddah, Saudi Arabia \\ ${ }^{2}$ Alnoor Specialist Hospital, Mecca, Saudi Arabia \\ ${ }^{3}$ Radiology Department, IMC Hospital, Jeddah, Saudi Arabia
}

Received: $18 / 10 / 2017$

Accepted: 25/10/2017

Published: 09/11/2017

How to cite this article: Khalil A, Bafaraj M, Badr B, Azzo M, Sabry A. An abdominal aortic aneurysm, intramural thrombus and moderate leak in an asian man presenting with acute gastroenteritis. EJCRIM 2017;4: doi:10.12890/2017_000759

Conflicts of Interests: The Authors declare that there are no competing interests.

Acknowledgements: Authors of IMC hospital in Jeddah Saudi Arabia would like to express their deepest gratitude to Alnoor Specialists Hospital in Makkah and Vascular Surgery team there. We would like to thank Dr Tamer Ahmed, Dr Roba Dahbol \& Dr Shireen Alsherief for their efforts too.

This article is licensed under a Commons Attribution Non-Commercial 4.0 License

\section{ABSTRACT}

Introduction: Missing a leaking abdominal aortic aneurysm (AAA) is common in medical practice because few at-risk patients have a history of AAA and many have an unusual presentation.

Background: AAA is less common among Asians than white Caucasians of the same age. Our patient had no significant risk factors apart from age and sex and had an unusual presentation.

Patient and Methods: A 67-year-old Asian man presented to the emergency room (ER) with a 1-day history of nausea, vomiting, diarrhoea, fever and abdominal pain. He was febrile, dehydrated. and had marked tenderness at the right iliac fossa. Laboratory findings suggested bacterial gastroenteritis but this did not explain the localized tenderness at the right iliac fossa.

Result and Discussion: A CT scan of the abdomen revealed an AAA arising above the origins of the renal arteries, an intramural thrombus, a retroperitoneal haematoma and a leak extending to the right iliac fossa. The patient was transferred to another hospital and underwent exploratory laparotomy, surgical repair of the aneurysm, and aortobi-iliac grafting with removal of the thrombus. The patient was discharged in good shape 3 weeks after surgery. Without the CT scan of the abdomen, the AAA could have been missed and the patient treated for severe gastroenteritis.

\section{LEARNING POINTS}

- Although abdominal aortic aneurysm leak is a rare differential diagnosis for elderly Asian patients with abdominal pain, it should be still suspected and investigated accordingly.

- In our elderly patient, as localized tenderness on clinical examination could not be explained by the gastroenteritis, an imaging study was essential to rule out a surgical cause.

\section{KEYWORDS}

Abdominal aortic aneurysm, ruptured aneurysm, unusual presentation, acute gastroenteritis, intramural thrombus 


\section{INTRODUCTION}

Most cases of abdominal aortic aneurysm (AAA) involve the segment between the renal arteries and the inferior mesenteric arteries (infrarenal), while approximately $5 \%$ involve the renal or visceral arteries ${ }^{[1]}$. The annual risk for bleeding is $0 \%$ for aneurysms less than $4 \mathrm{~cm}$ in diameter but $30-50 \%$ for those above $8 \mathrm{~cm}$ in diameter ${ }^{[2]}$. Of the $50 \%$ of patients with a ruptured AAA who reach hospital, $30-50 \%$ will die there ${ }^{[3]}$. In white Americans aged $65-80$ years, the prevalence of AAA is 4-8\% among males and $0.6-2 \%$ among females ${ }^{[4,5]}$. The incidence is 10 times lower among Asians, at $0.45 \%{ }^{[6]}$. Tobacco is a strong risk factor for developing AAA, as is systemic atherosclerosis. In contrast, diabetes mellitus has a negative association ${ }^{[7]}$. About $30 \%$ of cases are misdiagnosed as renal colic, acute coronary syndrome, perforated viscus, diverticulitis, gastrointestinal haemorrhage or bowel ischaemia ${ }^{[8]}$.

\section{CASE PRESENTATION}

A 67-year-old Asian man attended the emergency room (ER) of the IMC hospital in Jeddah with a 1-day history of nausea, vomiting, diarrhoea, fever and lower abdominal pain. He had no haematemesis, melena or urinary complaints but had passed eight watery bowel motions. He denied any previous febrile illness, sweating, weight loss or sexually transmitted disease (STD). His past medical, surgical and drug histories were unremarkable and he had no allergies. He was a businessman, a non-smoker and did not drink alcohol. He had come to Saudi Arabia to perform Umrah (Islamic pilgrimage to Mecca).

He looked sick with dry mucous membranes. His temperature was $38.7^{\circ} \mathrm{C}$, pulse rate was $112 / \mathrm{min}$ regular, blood pressure was $140 / 90$ and oxygen saturation was $98 \%$ in room air. He had severe tenderness at the right iliac fossa with no rebound. Bowels sounds were normal, and heart and chest examinations were unremarkable.

He had a white blood cell (WBC) count of 24,000×10\%/ (4,000-11,000×10\%/l) with 90\% neutrophils, haemoglobin $10 \mathrm{~g} / \mathrm{dl}$ (13.2-16.8 gm/dl) and normal mean cell volume (MCV) and platelets. Serum creatinine was $190 \mu \mathrm{mol} / \mathrm{I}$ (up to $106 \mu \mathrm{mol} / \mathrm{I}$ ) and urea nitrogen was $12.5 \mathrm{mmol} / \mathrm{I}$ (3.5-7.1 mmol/l), but the rest of the electrolytes and liver function tests were normal. His coagulation profile and lactic acid were normal. Stool analysis showed WBCs $>20 /$ high power field (hpf), red blood cells (RBCs) 3-5/hpf and no ova or parasites. Blood culture and stool culture came back negative after 72 hours. Clostridium difficile polymerase chain reaction (PCR) was negative. His $\mathrm{C}$-reactive protein was high at $70 \mathrm{mg} / \mathrm{l}(0-5 \mathrm{mg} / \mathrm{l})$.

The clinical picture and laboratory findings suggested acute gastroenteritis of bacterial origin, but the tenderness at the right iliac fossa was worrying. The patient received saline, paracetamol and ciprofloxacin $500 \mathrm{mg}$ intravenously.

A CT scan of the abdomen and pelvis (without intravenous contrast) revealed an AAA arising just above the origins of both renal arteries with a hyper-dense retroperitoneal haematoma (white arrow) and an intramural thrombus (Fig. 1). The AAA measured 74 mm at the maximum sagittal diameter (Fig. 2, white ruler)and an aortic leak was noted extending to the right psoas muscle and right iliac fossa (Fig. 3).The results were reported immediately to the ER physician.

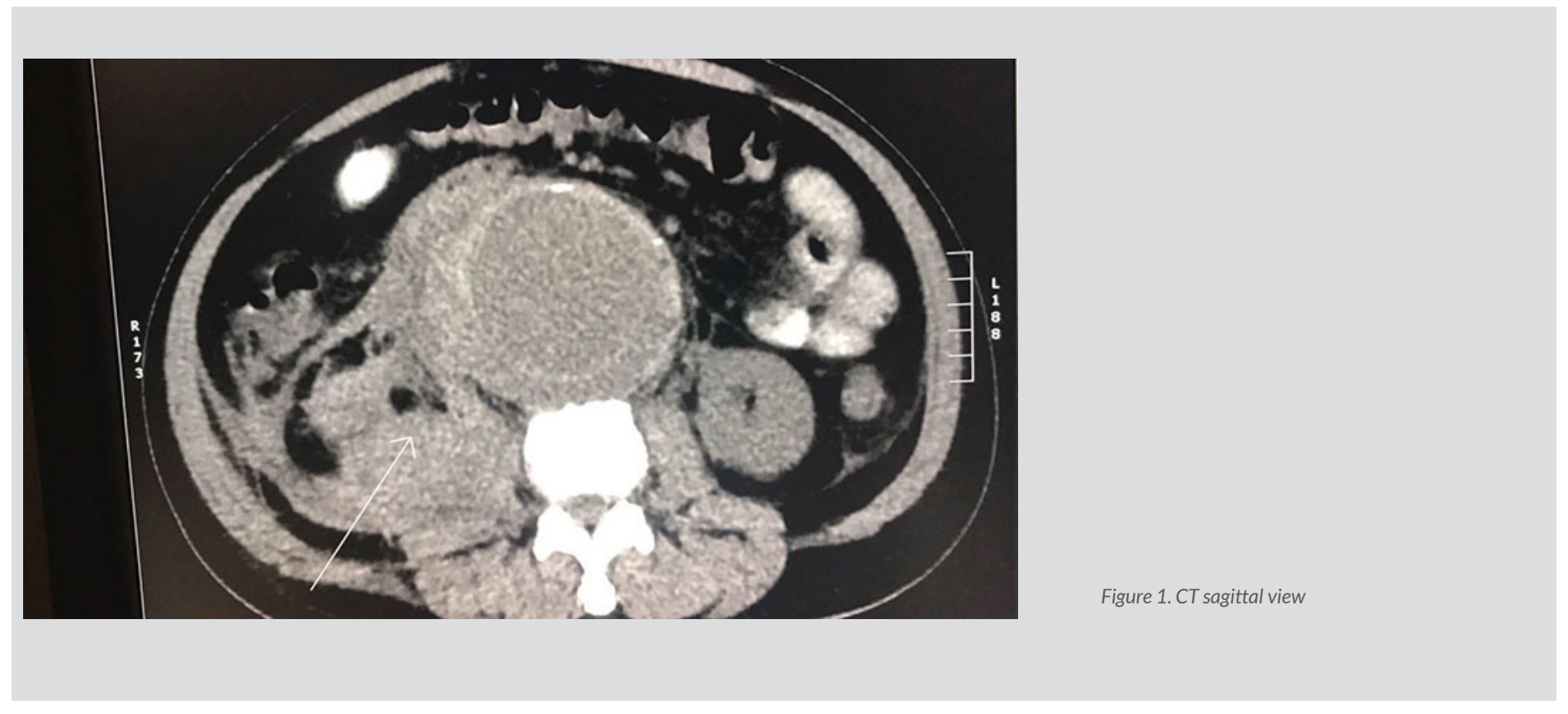




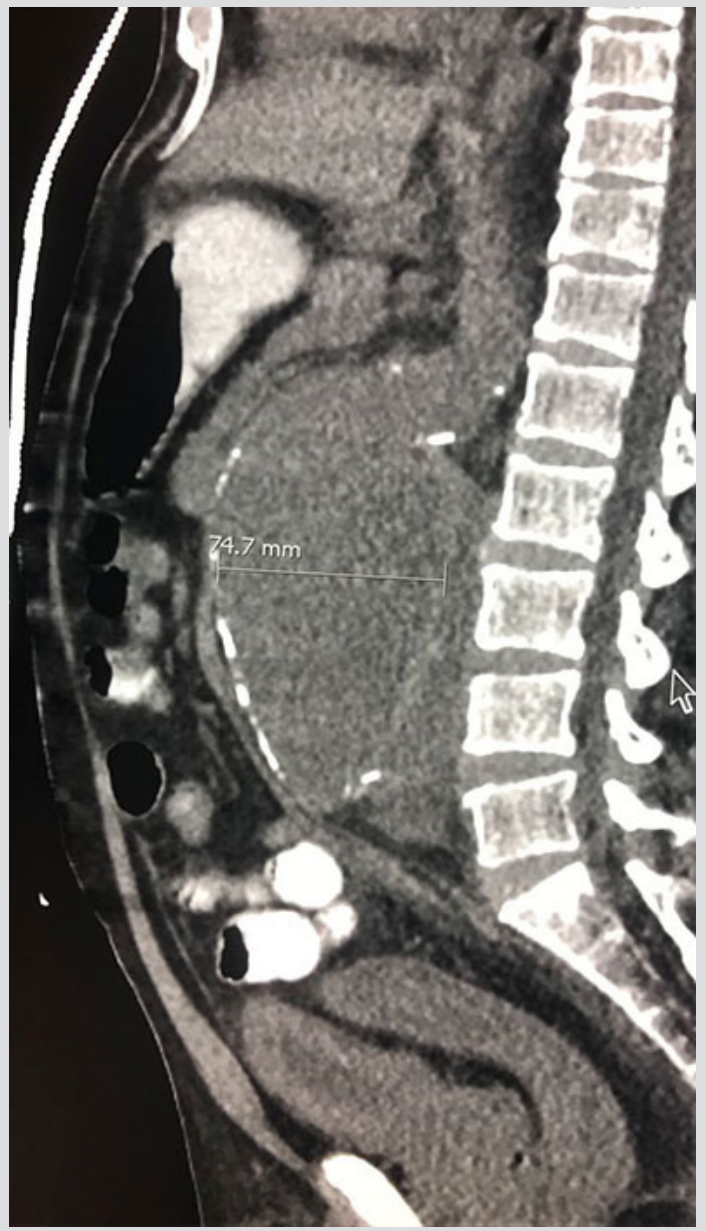

Figure 2. CT sagittal view

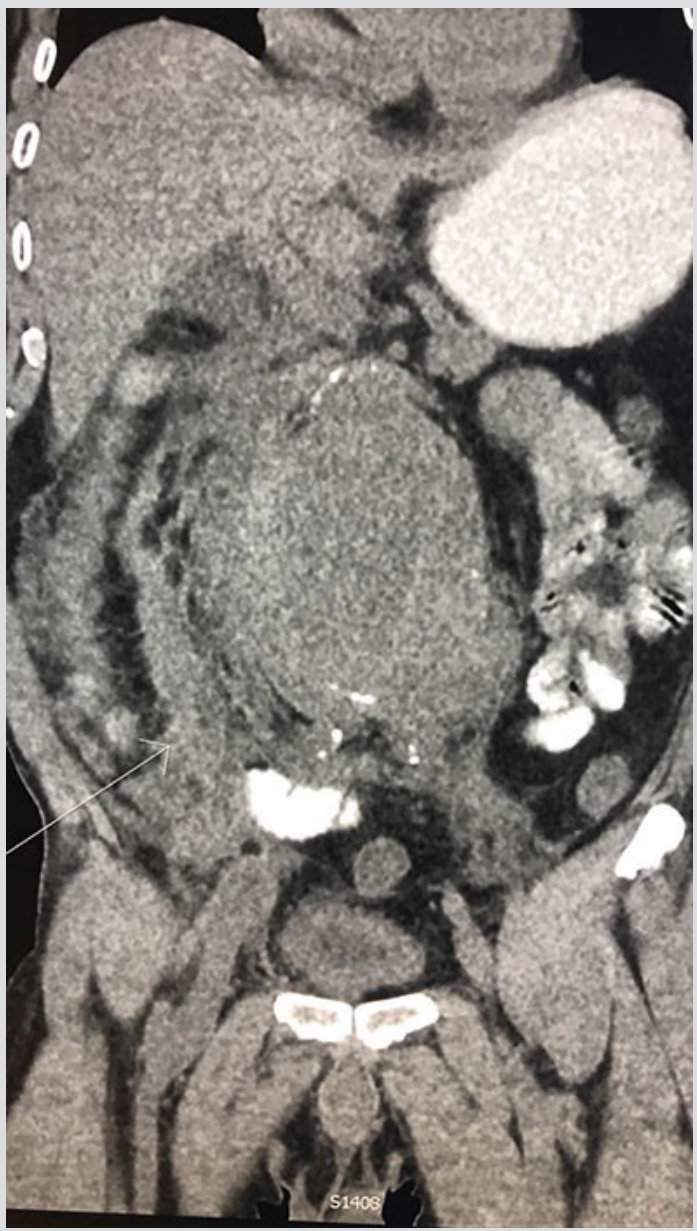

Figure 3. CT coronal view

Good equal bilateral arterial pulsations were seen in both upper and lower extremities. The patient was transferred to the vascular surgery team at Alnoor Hospital. Empirical antibiotics were continued, the patient's fever resolved and inflammatory markers improved significantly. Rapid plasma reagin for syphilis (RPR) serology and chest $x$-ray were normal. The patient underwent exploratory laparotomy and was found to have a large leaking juxtarenal AAA $10 \times 7 \mathrm{~cm}$ in diameter. The aneurysm extended from the level of the renal artery down to the level of the iliac bifurcation. The aneurysm was surgically repaired: an intramural thrombus was removed and a bifurcated silver-coated $20 \times 10 \mathrm{~mm}$ Dacron graft was applied.

Postoperatively, patient was moved to the intensive care unit (ICU) where he received ventilation, inotropes and correction of his anaemia. After 2 days, he was taken off the ventilator and inotropes. The patient's renal functions had normalized by the fifth postsurgical day, so he was transferred to a normal ward and started rehabilitation. He was discharged 3 weeks after surgery and flew back home.

\section{DISCUSSION}

Patients with a ruptured AAA presenting to the ER are a challenge for the assessing physician. Only $20-30 \%$ of such patients have known a history of $A A A^{[9]}$. Pain was the presenting symptom in $75 \%$ of 66 patients with a ruptured AAA: $45 \%$ had abdominal pain, $17 \%$ had flank/ back pain and $14 \%$ had both ${ }^{[10]}$. The unusual presentation of a ruptured AAA with hip pain only has been reported ${ }^{[11]}$. In one case, isolated testicular pain and a haematoma at the left iliopsoas muscle were the presenting symptoms ${ }^{[12]}$.

An electronic search identified one case report of a ruptured AAA of an iliac artery presenting with left iliac fossa pain and diarrhoea ${ }^{[13]}$. 
The only risk factors for AAA in our elderly Asian male patient were his age and sex. His preliminary assessment suggested acute bacterial gastroenteritis, but this could not have caused the tenderness at the right iliac fossa. However, the patient's CT scan showed a haematoma tracking to the right iliac fossa which explained the examination finding and revealed the AAA with an intramural thrombus. Infectious AAA is a rare differential diagnosis for patients with $A A A^{[8]}$. However, our patient had no history of previous febrile illness, his gastroenteritis was only a 1-day illness and his septic screen and RPR were negative.

If the CT scan of the abdomen and pelvis had not been carried out, the patient might have been admitted as having acute gastroenteritis and dehydration. Any future hypotension could have been wrongly explained as shock related to sepsis, which could have adversely affected management and outcome.

\section{REFERENCES}

1. Jongkind V, Yeung KK, Akkersdijk GJ, et al. Juxtarenal aortic aneurysm repair. J Vasc Surg 2010;52:760.

2. Brewster DC, Cronenwett JL, Hallett JW Jr, et al. Guidelines for the treatment of abdominal aortic aneurysms. Report of a subcommittee of the Joint Council of the American Association for Vascular Surgery and Society for Vascular Surgery. J Vasc Surg 2003;37:1106-1117.

3. Hoornweg LL, Storm-Versloot MN, Ubbink DT, et al. Meta analysis on mortality of ruptured abdominal aortic aneurysms. Eur J Vasc Endovasc Surg 2008;35:558.

4. Ashton HA, Buxton MJ, Day NE, et al. The Multicentre Aneurysm Screening Study (MASS) into the effect of abdominal aortic aneurysm screening on mortality in men: a randomised controlled trial. Lancet 2002;360:1531.

5. Ashton HA, Gao L, Kim LG, et al. Fifteen-year follow-up of a randomized clinical trial of ultrasonographic screening for abdominal aortic aneurysms. Br J Surg 2007:94:696.

6. Salem MK, Rayt HS, Hussey G, et al. Should Asian men be included in abdominal aortic aneurysm screening programmes? Eur J Vasc Endovasc Surg 2009;38:748.

7. Kent KC, Zwolak RM, Egorova NN, et al. Analysis of risk factors for abdominal aortic aneurysm in a cohort of more than 3 million individuals. $J$ Vasc Surg 2010;52:539.

8. Azhar B, Patel SR, Holt PJ, et al. Misdiagnosis of ruptured abdominal aortic aneurysm: systematic review and meta-analysis. J Endovasc Ther 2014;21:568.

9. Marston WA, Ahlquist R, Johnson G Jr, Meyer AA. Misdiagnosis of ruptured abdominal aortic aneurysms. J Vasc Surg 1992;16:17.

10. Rinckenbach S, Albertini JN, Thaveau F, et al. Prehospital treatment of infrarenal ruptured abdominal aortic aneurysms: a multicentric analysis. Ann Vasc Surg 2010;24:308.

11. Vaidyanathan S, Wadhawan H, Welch P, El-Salamani M. Ruptured abdominal aortic aneurysm masquerading as isolated hip pain: an unusual presentation. CJEM 2008;10:251.

12. Regus S, Apel H, Dankerl P, Lang W. Isolated testicular pain mimicking ruptured abdominal aortic aneurysm in a nonagenarian. Am J Emerg Med 2015;33:1117.e1-2.

13. Hartley E, Richards J. Isolated iliac artery aneurysm rupture presenting as left iliac fossa pain and diarrhea: a case report. Int J Surg Case Rep 2011;2:56-57. 